\title{
Schur finiteness and nilpotency
}

\author{
Alessio Del Padrone \\ delpadro@dima.unige.it \\ Carlo Mazza \\ carlo@math.ias.edu
}

July 5th, 2005

\begin{abstract}
Let $\mathcal{A}$ be a $\mathbb{Q}$-linear pseudo-abelian rigid tensor category. A notion of finiteness due to Kimura and (independently) O'Sullivan guarantees that the ideal of numerically trivial endomorphism of an object is nilpotent. We generalize this result to special Schur-finite objects. In particular, in the category of Chow motives, if $X$ is a smooth projective variety which satisfies the homological sign conjecture, then Kimura-finiteness, a special Schur-finiteness, and the nilpotency of $C H^{n i}\left(X^{i} \times X^{i}\right)_{n u m}$ for all $i$ (where $n=\operatorname{dim} X)$ are all equivalent.
\end{abstract}

Let $\mathcal{A}$ be a pseudo-abelian tensor category, i.e., a " $\otimes$-catégorie rigide sur $F "$ as in And04, 2.2.2] in which idempotents split. We have $F$-linear trace maps tr: $\operatorname{End}_{\mathcal{A}}(A) \longrightarrow \operatorname{End}_{\mathcal{A}}(\mathbb{1})$ compatible with $\otimes$-functors, and $F$-submodules of numerically trivial morphisms $\mathcal{N}\left(A_{1}, A_{2}\right):=\left\{f \in \operatorname{Hom}_{\mathcal{A}}\left(A_{1}, A_{2}\right)\right.$ $\operatorname{tr}(f \circ g)=0, \quad$ for all $\left.g \in \operatorname{Hom}_{\mathcal{A}}\left(A_{2}, A_{1}\right)\right\}$. We assume that $F=\operatorname{End}_{\mathcal{A}}(\mathbb{1})$ and it contains $\mathbb{Q}$. If $F$ is a field, $\mathcal{N}$ is the biggest non trivial $\otimes$-ideal of $\mathcal{A}$, and so it contains any morphism annihilated by some $\otimes$-functor.

Example 0.1 And04, Ch. 4] Assume $F$ is a field. For any admissible equivalence $\sim$ on algebraic cycles, motives of smooth projective varieties over a field $k$ with coefficients in $F$ form such a category $\mathcal{A}:=\mathcal{M}_{\sim}(k)_{F}$. If $X$ is a variety, we write $\mathfrak{h}(X)$ for its motive. For any $f \in \operatorname{End}_{\mathcal{A}}(\mathfrak{h}(X)), \operatorname{tr}(f)=\operatorname{deg}\left(\Gamma_{f} \cdot \Delta_{X}\right)$ and therefore $\mathcal{N}(\mathfrak{h}(X))=\mathcal{Z}_{\sim}^{\operatorname{dim}(X)}(X \times X)_{F, \text { num }}$ (numerically trivial correspondences of degree zero). If $\sim$ is finer than homological equivalence then any Weil cohomology $H$ factors through $a \otimes$-functor on $\mathcal{A}$, and $\operatorname{tr}(f)=\sum_{j}(-1)^{j} \operatorname{Tr}\left(f \mid H^{j}(X)\right)$ by the Lefschetz formula.

Recall that the partitions $\lambda$ of an integer $n$ give a complete set of mutually orthogonal central idempotents $d_{\lambda}:=\frac{\operatorname{dim} V_{\lambda}}{n !} \sum_{\sigma \in \Sigma_{n}} \chi_{\lambda}(\sigma) \sigma$ in the group algebra $\mathbb{Q} \Sigma_{n}$ (see [FH91). We define an endofunctor on $\mathcal{A}$ by setting $S_{\lambda}(A)=d_{\lambda}\left(A^{\otimes n}\right)$. This is a multiple of the classical Schur functor corresponding to $\lambda$. In particular, we define $\operatorname{Sym}^{n}(A)=S_{(n)}(A)$ and $\Lambda^{n}(A)=S_{\left(1^{n}\right)}(A)$. The following definitions are directly inspired by [Del02] and [Kim05] (see [AK02, GP03, and Maz04 for further reference). 
Definition 0.2 An object $A$ of $\mathcal{A}$ is Schur-finite if there is a partition $\lambda$ such that $S_{\lambda}(A)=0$. If $S_{\lambda}(A)=0$ with $\lambda$ of the form $(n)$ (respectively, $\lambda=\left(1^{n}\right)$ ) then $A$ is called odd (respectively, even). We say that $A$ is Kimura-finite if $A=A_{+} \oplus A_{-}$with $A_{+}$even and $A_{-}$odd.

Every Kimura-finite object is Schur-finite, but the converse fails, for example, in the category of super-representations of $G L(p \mid q)$. In Kim05, 7.5] and [AK02, 9.1.14] it was proven that if $A$ is a Kimura-finite object then the ideal $\mathcal{N}(A)$ is nilpotent.

In the case of example 0.1, an interesting consequence of the nilpotence of $\mathcal{N}(M)$ is that a summand $N$ of $M$ is zero if and only if its cohomology is zero (the idempotent defining $N$ must then be nilpotent). The nilpotency was used in [GP03, Theorem 7] to show the equivalence of Bloch's conjecture for a smooth projective suface $X$ with $p_{g}=0$ and the Kimura-finiteness of the motive of $X$, improving Kim05, 7.7].

Albeit in general Schur-finiteness is not sufficient to get the nilpotency of $\mathcal{N}(A)$ (see AK02, 10.1.1]), we will identify additional conditions which imply the nilpotency. In the category of motives we will show that for a motive which is Kimura-finite modulo homological equivalence, the Kimura-finiteness modulo rational equivalence is equivalent to the Schur-finiteness for a particular rectangle.

\section{A technical result}

Theorem 1.1 Suppose that $S_{\lambda}(A)=0$ for a partition $\lambda$ of $n \geq 2$ with $a_{\lambda}$ rows and $b_{\lambda}$ columns. Let $s:=a_{\lambda}+b_{\lambda}-1$ be the length of its biggest hook $\nu$, and $r:=n-s$. Assume that either $\lambda$ is a hook or that there is a $g \in \operatorname{End}_{\mathcal{A}}(A)$ with trace $t:=\operatorname{tr}(g)=\cdots=\operatorname{tr}\left(g^{\text {or }}\right)$, and $t \notin\left\{-\left(b_{\lambda}-2\right), \ldots, a_{\lambda}-2\right\}$. Then $f^{\circ(s-1)}=0$ for each $f \in \mathcal{N}(A)$, and so $\mathcal{N}(A)$ is nilpotent.

Proof 1 The last statement follows from [AK02, 7.2.8]: $\mathcal{N}(A)^{2^{s-1}-1}=0$.

For $\sigma \in \Sigma_{n}$, we index the corresponding decomposition of $\{1, \ldots, n\}$ into disjoint cycles $\gamma_{1}, \ldots, \gamma_{n}$ so that the support of $\gamma_{1}$ contains 1 ; moreover we define $l_{i}$ to be the order of the cycle $\gamma_{i}$, and $L=L(\sigma):=\max _{i}\left\{l_{i}\right\}$ to be the maximum length of the cycles of $\sigma$.

As $S_{\lambda}(A)=0$ we have $\sum_{\sigma} \chi_{\lambda}(\sigma) \cdot \sigma \circ f_{1} \otimes \cdots \otimes f_{n}=0$ for any $f_{1}, \ldots, f_{n} \in$ $\operatorname{End}_{\mathcal{A}}(A)$. By the Murnaghan-Nakayama rule (see [FH91, Problem 4.45]) $\chi_{\lambda}(\sigma)=$ 0 if $L(\sigma)>s$. Hence [AK02, 7.2.6] with $A_{1}=\cdots=A_{n}=A$, gives that in $\operatorname{End}_{\mathcal{A}}(A)$

$$
\sum_{\sigma \in \Sigma_{n}: L(\sigma) \leq s} \chi_{\lambda}(\sigma) \cdot t_{\sigma} \cdot f_{\gamma_{1}}=0
$$

where $f_{\gamma_{1}}:=f_{\gamma_{1}^{l_{1}-1}(1)} \circ \cdots \circ f_{\gamma_{1}(1)} \circ f_{1}, t_{\sigma}:=\prod_{j=2}^{q} t_{\sigma, j}$, and $t_{\sigma, j}:=\operatorname{tr}\left(f_{\gamma_{j}{ }^{l_{j}-1}\left(k_{j}\right)} \circ\right.$ $\cdots \circ f_{\gamma_{j}\left(k_{j}\right)} \circ f_{k_{j}}$ ) with $k_{j}$ any element in the support of $\gamma_{j}$ (if $l_{1}=n$, i.e. $q=1$, then $\left.t_{\sigma}=1\right)$.

Set $f_{1}:=\operatorname{Id}_{A}$ and $f_{2}=\cdots=f_{s}:=f$ (still no restrictions on $f_{s+1}, \ldots, f_{n}$ ). If $\operatorname{Supp}\left(\gamma_{1}\right) \subsetneq\{1, \ldots, s\}$, not all of the $f$ 's are in the composition $f_{\gamma_{1}}$, hence at 
least one of them must appear in a trace $\operatorname{tr}\left(f_{\gamma_{j}{ }^{l_{j}-1}\left(k_{j}\right)} \circ \cdots \circ f_{\gamma_{j}\left(k_{j}\right)} \circ f_{k_{j}}\right)$. But $f$ is numerically trivial, so $t_{\sigma}=0$ for any such $\sigma$, and

$0=\sum_{\sigma \in \Sigma_{n}: \operatorname{Supp}\left(\gamma_{1}\right)=\{1, \ldots, s\}} \chi_{\lambda}(\sigma) \cdot t_{\sigma} \cdot f_{\gamma_{1}}=\left(\sum_{\sigma \in \Sigma_{n}: \operatorname{Supp}\left(\gamma_{1}\right)=\{1, \ldots, s\}} \chi_{\lambda}(\sigma) \cdot t_{\sigma}\right) f^{\circ(s-1)}=x \cdot f^{\circ(s-1)}$,

where $x:=\sum_{\sigma \in \Sigma_{n}: \operatorname{Supp}\left(\gamma_{1}\right)=\{1, \ldots, s\}} \chi_{\lambda}(\sigma) \cdot t_{\sigma} \in F$. It is enough to show $x \neq 0$ for some choice of the $f_{i}$ 's.

If $r=0$ then $\lambda=\nu=\left(n-j, 1^{j}\right)$ is itself a hook, $t_{\sigma}=1$ for any $\sigma$ with $l_{1}=n$ and by [FH91, Exercise 4.16] $x$ is just $(n-1) !(-1)^{j} \neq 0$, hence $\mathcal{N}(A)$ is nilpotent.

If $\lambda$ is not a hook let $\delta:=\lambda \backslash \nu$. The element $x \in F$ is a sum over $\sigma=\gamma_{1} \circ \sigma^{\prime}$ such that $\gamma_{1}$ is an s-cycle of $\{1, \ldots, s\}$ and $\sigma^{\prime}$ is a permutation of $\{s+1, \ldots, n\}$, so by Murnaghan-Nakayama $\chi_{\lambda}(\sigma)=\chi_{\lambda \backslash \nu}\left(\sigma^{\prime}\right)$, and $x=(-1)^{a_{\delta}-1} \mid\left\{s-\right.$ cycles of $\left.\Sigma_{n}\right\} \mid \sum_{\sigma^{\prime} \in \Sigma_{r}} \chi_{\delta}\left(\sigma^{\prime}\right) \cdot t_{\sigma}$. Thus we are reduced to study elements of the form

$$
y\left(\delta ; g_{1}, \ldots, g_{r}\right):=\sum_{\sigma \in \Sigma_{r}} \chi_{\delta}(\sigma) \cdot \prod_{j=1}^{q} t_{\sigma, j}
$$

where we can choose freely $g_{1}, \ldots, g_{r} \in \operatorname{End}_{\mathcal{A}}(A)$.

Take $g \in \operatorname{End}_{\mathcal{A}}(A)$ as in the hypothesis, then $y(\delta ; g, \ldots, g)=\sum_{\sigma \in \Sigma_{r}} \chi_{\delta}(\sigma)$. $t^{\mid c y c l e s}$ of $\sigma \mid$ is the polynomial in $t=\operatorname{tr}(g)$ called the content polynomial of $\delta$. It decomposes as $y(\delta ; g)=\chi_{\delta}\left(\operatorname{Id}_{\Sigma_{r}}\right) \cdot \prod_{(i, j) \in \delta}(t+j-i)$, then $y(\delta ; g)=0$ if and only if $\operatorname{tr}(g) \in\left\{-\left(b_{\delta}-1\right), \ldots, a_{\delta}-1\right\} \subseteq\left\{-\left(b_{\lambda}-2\right), \ldots, a_{\lambda}-2\right\}$. By hypothesis, there is a $g$ such that $y(\delta ; g) \neq 0$, which implies that $x \neq 0$, which in turn implies that $f$ is nilpotent. Hence the theorem is proven.

Remark 1 (B. Kahn) The existence of a $g \in \operatorname{End}_{\mathcal{A}}(A)$ with $\operatorname{tr}(g) \neq 0$ is not enough to ensure the nilpotency of $\mathcal{N}(A)$ with A Schur-finite. In [AK02, 10.1.1] it is exhibited a non-zero Schur-finite object $A^{\prime}$ with $\mathcal{N}\left(A^{\prime}\right)=\operatorname{End}_{\mathcal{A}}\left(A^{\prime}\right)$ : it suffices to look at $A:=A^{\prime} \oplus \mathbb{1}^{n}$.

Conjecture 1 From numerical evidence ([GAP04]) we conjecture a stronger version of Theorem 1.1. Let $A$ be an object with two endomorphisms $\pi_{1}$ and $\pi_{2}$ such that $a:=\operatorname{tr}\left(\pi_{1}\right)=\operatorname{tr}\left(\pi_{1}^{\circ i}\right)$ for all $i, b:=\operatorname{tr}\left(\pi_{2}\right)=\operatorname{tr}\left(\pi_{2}^{\circ j}\right)$ for all $j$, and $\operatorname{tr}\left(\pi_{1}^{\circ i} \circ \pi_{2}^{\circ j}\right)=0$ for all $i$ and $j$. If $S_{\lambda}(A)=0$ where $\lambda \not \supset(b+2)^{a+2}$, then $y\left(\lambda \backslash \nu ; \alpha_{1} \pi_{1}+\alpha_{2} \pi_{2}\right) \neq 0$ (as a polynomial in $\alpha_{1}$ and $\alpha_{2}$ ) and hence $\mathcal{N}(A)$ is nilpotent.

\section{Motives and nilpotency}

Let now $\mathcal{A}$ be the category of Chow motives $\mathcal{M}_{\text {rat }}(k)_{\mathbb{Q}}$ (example 0.1), let $H$ be any Weil cohomology, and let $X$ be a smooth projective variety. The cohomology $H(X)$ is a super vector space of dimension $\left(d_{e v}, d_{o d d}\right)$, and we set $\lambda_{H(X)}:=$ $\left(\left(d_{o d d}+1\right)^{d_{e v}+1}\right)$ (the rectangle with $d_{o d d}+1$ columns and $d_{e v}+1$ rows). By [Del02, 1.9], $S_{\lambda}(H(X)) \neq 0$ if and only if $\lambda \not \supset \lambda_{H(X)}$. Hence, $S_{\lambda}(\mathfrak{h}(X)) \neq 0$ if $\lambda \not \supset \lambda_{H(X)}$. So $S_{\lambda}(\mathfrak{h}(X))=0$ implies that $\lambda \supset \lambda_{H(X)}$. 
Recall the "homological sign conjecture" (due to Jannsen, see And04, 5.1.3]): we say that $X$ satisfies the conjecture $C^{+}(X)$ if the projections on the even and the odd part of the cohomology are algebraic. This conjecture is stable under products, and it holds true, with respect to classical cohomologies, for abelian varieties and smooth projective varieties of dimension at most two. It can be shown that $C^{+}(X)$ is equivalent to the Kimura-finiteness of the motive of $X$ modulo homological equivalence.

Proposition 2.1 Let $X$ be a smooth projective variety, and let $\lambda$ be a partition with at most $d_{e v}+1$ rows or $d_{o d d}+1$ columns. If $S_{\lambda}(\mathfrak{h}(X))=0$ (and hence $\left.\lambda \supset \lambda_{H(X)}\right)$ and $C^{+}(X)$ holds, then $\mathcal{N}(\mathfrak{h}(X))$ is nilpotent. Moreover, if $X$ is a surface with $p_{g}=0$, Bloch's conjecture holds for $X$.

Proof $2 B y C^{+}(X)$ there are two cycles $\pi_{+}$and $\pi_{-}$inducing the projections on the even and odd cohomology. Then $d_{e v}=\operatorname{tr}\left(\pi_{+}\right)=\operatorname{tr}\left(\pi_{+}^{\circ i}\right)$ for all $i$, and $-d_{\text {odd }}=\operatorname{tr}\left(\pi_{-}\right)=\operatorname{tr}\left(\pi_{-}^{\circ j}\right)$ for all $j$. Then either $\pi_{+}$or $\pi_{-}$satisfies the condition of Theorem 1.1, and therefore $\mathcal{N}(\mathfrak{h}(X))$ is nilpotent. Bloch's conjecture is now a formal consequence of [Kim05, 7.6 and 7.7].

Theorem 2.2 Let $X$ be a smooth projective variety. Under $C^{+}(X)$ the following are equivalent:
1) $\mathfrak{h}(X)$ is Kimura-finite;
2) $S_{\lambda_{H(X)}}(\mathfrak{h}(X))=0$;
3) $\mathcal{N}\left(\mathfrak{h}\left(X^{n}\right)\right)$ is nilpotent for all $n \geq 1$.

Proof 3 It is easy to show that $1 \Rightarrow 2$. For $3 \Rightarrow 1$ we proceed as follows. As $C^{+}(X)$ holds and $\mathcal{N}(\mathfrak{h}(X))$ is nilpotent, then there exist two motives $X_{+}$ and $X_{-}$whose cohomologies are exactly the even and the odd part of $H(X)$. It is now easy to prove that $\mathfrak{h}(X)=M_{+} \oplus M_{-}$with $M_{+}$even and $M_{-}$odd because it will be enough to check it in cohomology. We need to verify $2 \Rightarrow 3$. Assume that $S_{\lambda_{H(X)}}(\mathfrak{h}(X))=0$. From the proof of [Del02, Cor. 1.13], we find that $S_{\lambda_{H\left(X^{n}\right)}}\left(\mathfrak{h}\left(X^{n}\right)\right)=S_{\lambda_{H\left(X^{n}\right)}}\left(\mathfrak{h}(X)^{\otimes n}\right)=0$. Since $C^{+}\left(X^{n}\right)$ holds true, Proposition 2.1 gives that $\mathcal{N}\left(\mathfrak{h}\left(X^{n}\right)\right)$ is nilpotent.

If Conjecture 1 is true, then Bloch's conjecture holds for any smooth projective surface $X$ with $p_{g}=0$ such that $S_{\lambda}(\mathfrak{h}(X))=0$ for $\lambda \not \supset\left(d_{\text {odd }}(X)+\right.$ $2)^{d_{e v}(X)+2}$.

\section{Acknowledgments}

This article was inspired by the preprint Gul by V. Guletskil. Both authors would like to thank the Institut de Mathématiques de Jussieu for hospitality while part of this manuscript was written. For financial support, the first author would like to thank the Fondazione Carige and the second author would like to thank Sergio Serapioni (Honorary President of the Società Trentina Lieviti, Trento, Italy).

\section{References}

[AK02] Y. André and B. Kahn, Nilpotence, radicaux et structures monoïdales, Rend. Sem. Mat. Univ. Padova 108 (2002), 107-291, with an appendix by P. O'Sullivan. MR 1956434 
[And04] Y. André, Une introduction aux motifs (motifs purs, motifs mixtes, périodes), Panoramas et Synthèses [Panoramas and Syntheses], vol. 17, Société Mathématique de France, Paris, 2004. MR MR2115000

[Del02] P. Deligne, Catégories tensorielles, Mosc. Math. J. 2 (2002), no. 2, 227-248, Dedicated to Yuri I. Manin on the occasion of his 65 th birthday. MR 1944506

[FH91] W. Fulton and J. Harris, Representation theory, Graduate Texts in Mathematics, vol. 129, Springer-Verlag, New York, 1991, A first course, Readings in Mathematics. MR 93a:20069

[GAP04] The GAP Group, GAP - Groups, Algorithms, and Programming, Version 4.4, 2004, (http://www.gap-system.org).

[GP03] V. Guletskiu and C. Pedrini, Finite-dimensional motives and the conjectures of Beilinson and Murre, K-Theory 30 (2003), no. 3, 243-263, Special issue in honor of Hyman Bass on his seventieth birthday. Part III. MR MR2064241

[Gul] V. Guletskiŭ, A remark on nilpotent correspondences, Preprint, January 27, 2004, K-theory Preprint Archives, http://www.math.uiuc.edu/K-theory/0651/.

[Kim05] S.-I. Kimura, Chow groups are finite dimensional, in some sense, Math. Annalen 331 (2005), no. 1, 173-201.

[Maz04] C. Mazza, Schur functors and motives, K-Theory 33 (2004), no. 2, 89-106. 